\title{
GCU
}

Glasgow Caledonian

University

University for the Common Good

\section{Low complexity object detection with background subtraction for intelligent remote monitoring}

\author{
Nazir, Sajid; Hamdoun, Hassan; Kaleem, Muhammad
}

Published in:

2019 International Conference on Information Science and Communication Technology, ICISCT 2019

DOI:

$10.1109 /$ CISCT.2019.8777413

Publication date:

2019

Document Version

Author accepted manuscript

Link to publication in ResearchOnline

Citation for published version (Harvard):

Nazir, S, Hamdoun, H \& Kaleem, M 2019, Low complexity object detection with background subtraction for intelligent remote monitoring. in 2019 International Conference on Information Science and Communication Technology, ICISCT 2019. IEEE, pp. 6-11, 2019 International Conference on Information Science and Communication Technology (ICISCT 2019), Karachi, Pakistan, 9/03/19.

https://doi.org/10.1109/CISCT.2019.8777413

\section{General rights}

Copyright and moral rights for the publications made accessible in the public portal are retained by the authors and/or other copyright owners and it is a condition of accessing publications that users recognise and abide by the legal requirements associated with these rights.

Take down policy

If you believe that this document breaches copyright please view our takedown policy at https://edshare.gcu.ac.uk/id/eprint/5179 for details

of how to contact us. 


\section{Low Complexity Object Detection with Background Subtraction for Intelligent Remote Monitoring}

\author{
Sajid Nazir \\ School of Engineering and Built \\ Environment \\ Glasgow Caledonian University \\ Glasgow, UK, G4 OBA \\ sajid.nazir@gcu.ac.uk
}

\author{
Hassan Hamdoun \\ BT Technology, Applied Research \\ British Telecommunications plc \\ Adastral Park, Suffolk, UK, IP5 3RE \\ hassan.hamdoun@bt.com
}

\author{
Mohammad Kaleem \\ Department of Electrical Engineering \\ COMSATS University \\ Islamabad, Pakistan \\ mkaleem@comsats.edu.pk
}

\begin{abstract}
Advancements in digital technologies have enabled cost-effective deployments of visual sensor nodes that can detect a motion event in the coverage area. The real world field remote monitoring image capture conditions, for example, in security and ecological studies are affected by wind, rain, snow, sunlight etc. and are seldom ideal. Motion detection is a precursor to subsequent intelligent processing on the image to extract information. Less complex object detection techniques often rely on maintaining a background image and subtracting foreground image, purporting to have an object in it, to create a difference image to determine the presence of a moving object. Correct object detection is critical as otherwise resulting false positive (without a moving object) images needlessly invoke further processing, storage and analysis. In this paper, we review background subtraction techniques and propose an image differencing technique that can significantly reduce the algorithm complexity along with other associated advantages. The results of proposed reduced image subset are provided to highlight the benefits.
\end{abstract}

Keywords-JPEG, image differencing, false positive, object detection, low complexity, ecology, computer vision

\section{INTRODUCTION}

The miniaturisation of embedded devices has resulted in bringing down the cost of development and deployment of visual sensor nodes together with increased processing power and on-board memory. These devices are deployed in the field conditions to run autonomously on small batteries for surveillance and remote monitoring [1].

We define intelligent remote monitoring as a system in which the embedded processor with an image acquisition system can autonomously detect a moving object and communicate the event by raising an alarm. A generic model for a motion detection system is shown in Figure 1. We assume a static camera system which operates autonomously and captures an image based on a motion trigger. The device also has access to a previous image from a continuous running video capture, a background model, or a time lapse capture.

Accurate and timely object detection is of paramount importance in surveillance and monitoring operations. A basic background and foreground difference image can detect changed pattern or the foreground object [2]. Many organisms use motion detection in visual processing and it is an important step in artificial vison systems [3]. If the system incorrectly perceives an event then it is known as a false positive and in excessive quantities unnecessarily overloads the system and causes operator fatigue. In field deployments, the camera operation relies on the battery power and must use it judiciously to enhance the deployment period. Thus it is important to determine 'motion' correctly before a

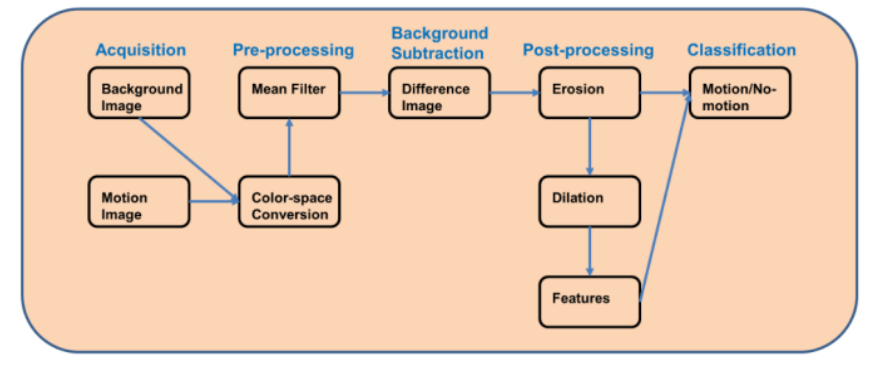

Fig. 1. Basic steps for a background subtraction process

processor-intensive operation such as video capture or communication is invoked.

The trigger for an image capture could be based on changes to a physical phenomenon such as heat, vibration, sound or physical activity such as an open door latch. In general the object detection techniques depend to some extent on a threshold. Thus, a false positive image that does not have an moving object, could be due to the equipment malfunction, environmental conditions (e.g., Passive Infrared being triggered due to a hot gust of air) [4] or a high/low threshold. The problem is compounded for outdoor environments which are much more challenging compared to indoor conditions.

This paper has the following contributions (1) provides a survey of background subtraction for object detection, (2) proposes using only image DC coefficients (Discrete Cosine Transform (DCT) coefficient with $8 \times 8$ sub-block average value) for reduction in algorithm complexity which can also aid correct detection, and (3) proposes use of simple features extracted from the difference image for improving detection.

Rest of the paper is organized as follows: Section II provides a survey of background subtraction techniques. An image differencing technique based on DC coefficients is proposed in Section III. The system model is described in Section IV. Results and discussion are provided in Section V and finally the conclusion in Section VI.

\section{BACKGROUND SUBTRACTION}

Background Subtraction is a useful but sensitive method for grey level differences in co-registered images [2]. Motion detection has a fundamental role in any monitoring or surveillance algorithm [5][6][7][8].

Image differencing or background subtraction classifies pixels into background representing static information and foreground pixels that represent moving objects [9]. It is a simple and robust image segmentation technique [10] with 
availability of static background image. It is largely useful in rigid background and similar lighting conditions [9][11]. Image difference does not depend on the local or global information in the image but rather on the grey levels [12].

A comprehensive discussion on the background subtraction method is given in [9]. A comprehensive review and comparison of twenty-nine background subtraction algorithms is provided in [13] using BMC (Background Models Challenge) dataset [14]. Benchmarking of the methods shows that simple static background methods have minimum memory and execution time [13]. A survey of different motion detection techniques in static background has been provided in [5].

Pre-processing through histogram matching that matches the maximum and minimum values of the histograms was used in [15] to improve the results. A technique based on using reference pixels to improve the results of frame differencing is proposed in [10]. The error in pixel classification can be reduced by considering the values of neighbouring pixels. Results are provided for two video sequences with 50 static background images and a single foreground image. The frame differencing was applied after averaging the 50 background images [10].

A method primarily reported for landslide detection, based on local mutual information and thresholding is described in [12] which is suitable for detection of large changed regions. The authors used connected component analysis on the blobs to identify the change detection [12]. Here, a blob means a set of pixels that are identified as part of the moving object as a result of image differencing. A qualitative and quantitative comparison of frame differencing and Gaussian mixture is provided by [16] for detection of birds under varying conditions in a video stream.

To overcome the difficulty of detecting motion by just one method, a real-time combination of information from structure and colour using census transform is proposed [6]. A low computation background subtraction with a background model where pixel grayscale information and colour invariant $\mathrm{H}$ are jointly used is described in [9]. The background model is updated based on pixels in modelled background and historical frames [9].

Background subtraction has been applied for motion detection in presence of camera jitter of an unstable camera [7]. They propose motion detection based on dynamics instead of colour or intensity by utilizing two thresholds which can also be calculated automatically. An efficient hardware based implementation of an image detection based on background subtraction, EBSCam has been described by [17]. A modified background subtraction method has been proposed in [8] claiming to address the ghosting and moving leaves problem.

\section{A. Simplified Differencing Schemes}

Simplified background subtraction just uses the previous frame, time lapse image or a background model to determine the object. A low complexity design for hardware implementation for low power devices is provided in [9]. Although, simplified image differencing would need only the closest previous frame, many techniques use a number of frames to model the background [9].

1) Double Difference: In order to improve the basic image differencing, double difference technique is sometimes used [18]. Double differences of the current image from the previous and next image frame can be combined using a logical AND operator [5].

2) Triple Difference: Background subtraction was combined using logical AND with three-frame difference to detect moving objects from underwater video in [19] by increasing the interval for frame differencing to four. Morphological processing was applied to eliminate the background noise.

3) Quadruple Difference: A technique based on frame differencing with four frames for motion detection in outdoor scenes is described in [8]. The scheme decreased the false alarm rate and used two thresholds.

\section{B. Reduced Complexity Operations}

Some computation intensive schemes that have higher accuracies may not be suitable for low power and smart camera applications [9].

A single day image differencing technique to identify animals across eight pastures using aerial imagery is described in [20] using Principal Component Analysis (PCA). PCA reduced the image dimensionality and provided the correlated information from the three bands. Uniform backgrounds such as sandy beaches, ice flows or oceans provided for easy differentiation of animals [20]. However, it could be argued that this would apply for animals which have sufficient colour difference from these uniform backgrounds.

A computationally efficient pixel-level change detection framework with a stationery camera with sigma-delta for background estimation is proposed by [21]. A limitation of the method was related to adaptation complexity of some complex scenes and that slow motion could be taken as a background.

In ecological applications, the importance of automatic image-based tracking is described in [11]. This avoids manual intervention and transforms the subjective decision making of a human to an objective comparison criteria [11].

A semi-automated system, AnimalFinder [22] is developed for animal detection in camera trap time lapse images to identify the presence of animal and to differentiate between white-tailed deer, wild pigs, and raccoons. The performance varied due to the setting of the threshold, however there was significant reduction to the images requiring manual review. A mean filter followed by a Canny edge detector was applied, identifying areas as 'blobs' which could be analysed for size and shape. The performance was checked for the threshold range from 0.01 to 0.95 .

A system for autonomous monitoring for cliff nesting seabirds is proposed in [23] using the time lapse images. The aim of study was to automatically estimate bird population. The challenges of outdoor natural environment are also discussed that contribute to generate false positives. A mixture of Guassian (MoG) based method in 5 dimensional feature space is proposed. However, the algorithm is computation intensive which makes it difficult even for low resolution images to run in real time, such that segmentation of an image on a general PC could take around a minute. However, this limitation does not hinder use of time lapse images [23]. 
A simplified image differencing scheme was implemented on a Raspberry Pi for the Wireless Internet Sensing Environment (WiSE) project [4] by the author. Following the steps shown in Figure 1, and using an automatically calculated threshold, combined with blob count and size, the motion activations were correctly classified [4]. An image recognition system was designed to identify avian activity using frame differencing and image segmentation making use of centroid and bounding boxes to validate the detection and location of a bird [16].

\section{Significance of Threshold}

The classification of a pixel into a blob is dependent on the relative difference between that position pixel in the motion activated and background image.

Threshold estimation is a difficult process [10]. A low threshold can incorrectly classify background as objects whereas a high threshold will classify real objects as background. Selection of an appropriate threshold based on image characteristics as explained below can also offset wrong classification of images.

A single global threshold could be a problem for frame differencing as the difference could be affected by the camera noise [10]. Threshold if set on a local adaptive threshold can give better results for false alarms, compared to a global threshold [24]. An adaptive automatic threshold algorithm is mentioned in [10]. Several image difference threshold strategies were investigated and hysteresis thresholding was shown to provide good results [2]. Computational complexities of the considered methods are also provided. The study [20] for identifying animals in aerial images used a semi-automated process as no viable automatic spatial or spectral threshold values could be determined.

\section{Proposed DC CoEfFicient BASEd Processing}

In this Section we propose a reduction in the algorithm complexity by restricting processing to DC coefficients only. This is a generic mechanism which is applicable to all the background subtraction processes described in Section II.

Although image differencing is a simple technique, it produces a large image matrix [12]. Restricting object detection analysis to DC coefficients reduces the image data to be processed. Scene changes in compressed video sequences using Discrete Cosine Transform (DCT) DC Coefficients and motion vectors without decoding the video is described in [24].

\section{A. JPEG Compression}

Joint Photographic Expert Group (JPEG) [25] image is the most prevalent image format in use. It's the de facto standard for still image capture and also is the preferred format for video cameras (as Motion JPEG) for security applications as it is easier to locate a frame in the compressed video compared to other true (inter frame) video compression standards like H.264 [26].

\section{B. Compressed Bitstream}

1) Header: JPEG header is followed by information about the image size, compression tables etc, which is followed by the compressed bitstream containing image data comprising DC and AC coefficients (at most 63).
2) DC Coefficients: These comprise the average value of the $8 \times 8$ sub-block. The main visual content of the image can be captured through just the DC coefficients.

3) AC Coefficients: These follow the DC coeffcient and represent the finer details in the image. If all 64 pixel values in $8 \times 8$ image block are same then its compressed representation will only have a DC value with all $\mathrm{AC}$ coefficients as zero.

\section{DC Extraction}

The visual sensors can capture images in large resolutions. Reducing the image size by deleting alternate rows and columns is a computationally expensive process. In comparison, DC coefficient extraction only requires simple parsing operation on the compressed bit stream.

\section{DC Only Benefits}

Utilizing only DC coefficients for further processing has the following advantages:

1) Noise Suppression: Sensor noise contributes significantly to the image differencing even between images that are very similar [15]. The intensity changes could also be due to camera noise or illumination differences [8]. The images under the same environmental conditions could be slightly different due to noise during image capture. The average value of the 64 pixels (DC coefficient) in the subblock reduces any spurious pixel value.

2) Reduction of Algorithm Complexity: Taking the DC coefficients alone reduces the pixel data to be processed. This reduces complexity of any algorithm that is processing the pixel data.

3) Reduction of Image Size: Taking the DC coefficients alone reduces the image size. This is much easier size reduction compared to e.g., by dropping alternate rows and columns which also does not provide comparable results.

4) Effects on object: This would also reduce the object size as $8 \times 8$ pixel blocks in the original image are now represented by a single DC value. We need to maintain a meaningful representation of objects and do not want them to disappear. Our results show that this is not the case for even small size objects in spite of 64 times reduction in image size for a DC only image.

\section{SYSTEM MODEL}

We implement image differencing as illustrated in Figure 1. An image differencing system would operate only on the DC image and optionally a Region-of-Interest (RoI) could also be selected. This keeps the system complexity low by discarding the finer details represented by high frequency DCT (AC) coefficients.

Let the background image be represented by $I_{B(x, y)}$ and the motion-activated image as $I_{M(x, y)}$, then the difference image, $I_{D(x, y)}$ will be represented as given in equation 1 .

$$
I_{D(x, y)}=I_{M(x, y)}-I_{B(x, y)}
$$

$I_{B(x, y)}$ will be totally black if there is no significant difference, but an object such as bird or animal present in motion-activated image, will appear as a white blob signifying the difference between pixel values. 


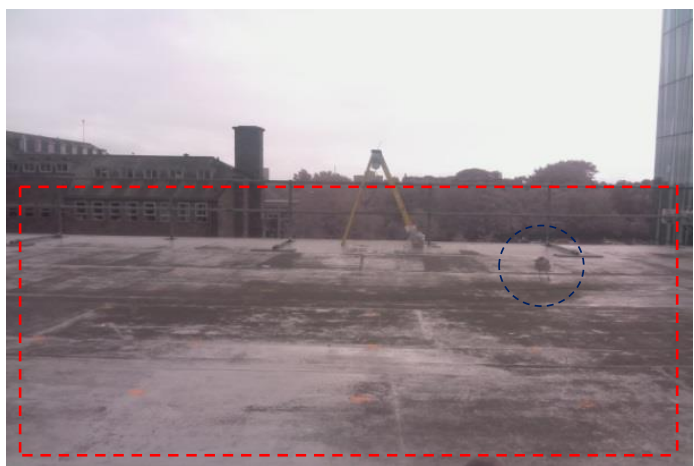

Fig. 2. A motion activated image.

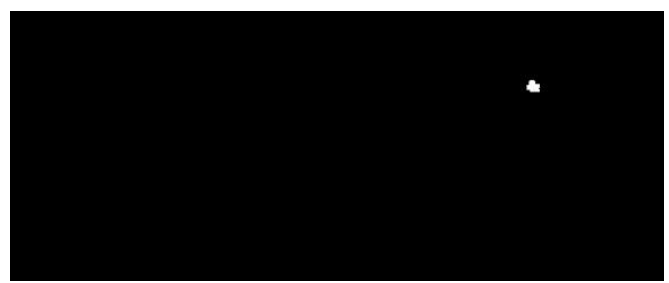

Fig. 3. A difference image subtracting Figure 2 from static image..

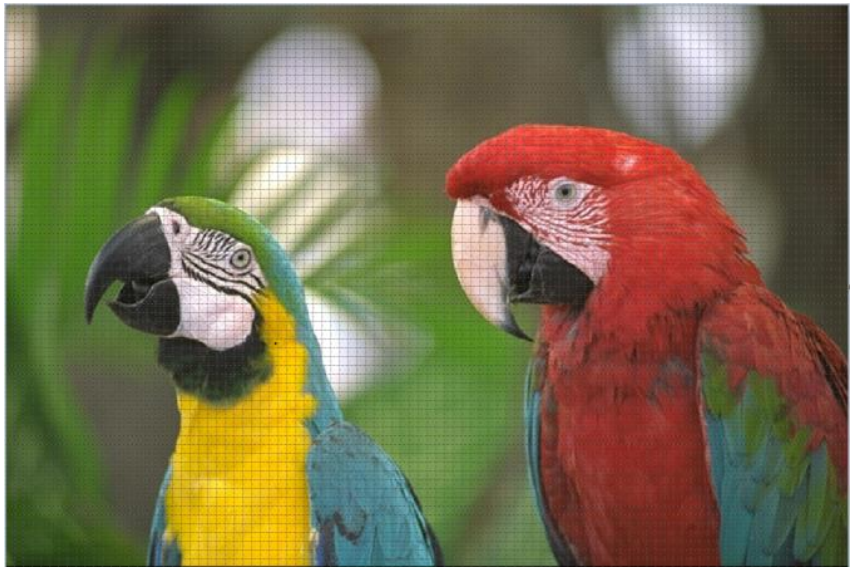

Fig. 4. Source Parrots image, resolution 768x512, with $8 \times 8$ grid superimposed to highlight the JPEG $8 \times 8$ sub-blocks.

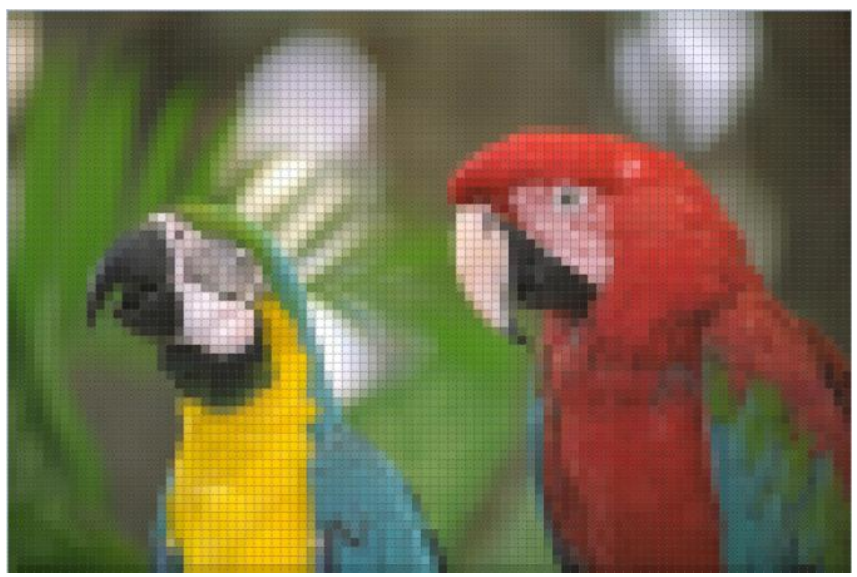

Fig. 5. Parrots image reconstructed from 96x64 DC Coefficients to recreate a 768x512 image. Each pixel grid block contains one DC coefficient value reproduced 64 times.

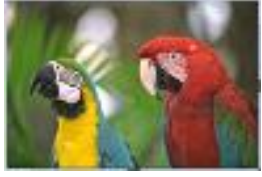

(a)

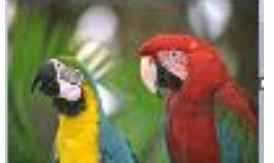

(b)
Fig. 6. Reduced size Parrots image (a) Using only DC coefficients, 17,431 bytes (b) Original image, 124,439 bytes.

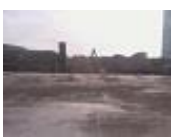

(a)

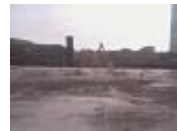

(b)

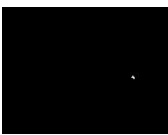

(c)
Fig. 7. DC Image Differencing Operation (a) Reduced static image (b) Reduced motion-activated image (from Fig 5) (c) DC Difference image.

Two images were selected for application of proposed methods. Firstly, we consider images (Figure 2 and 3) from public data repository for the WiseEye project [27] which has the background time lapse, motion-activated and difference images. The images are of low resolution and contrast making the task more challenging. We selected a motion-activated image from the WiseEye repository that had a bird present in the image after motion activation, shown as blue dashed circle in Figure 2, and the resulting difference image (Figure 3) for the selected Region of Interest (RoI). We also use publicly available parrots image (Figure 4) on which we have superimpose $8 \times 8$ pixel grid to highlight JPEG DCT processing.

\section{RESULTS AND DISCUSSION}

\section{A. DC Images}

The DC image for the parrots image is shown in Figure 5. It appears coarse-grained and the blockiness is due to a single pixel value (DC coefficient) now replacing all 64 pixels from the $8 \times 8$ image block. We could also view this image at a reduced size, that is, by using only the DC coefficient (average of 64 values) at a resolution of 96x64, as shown in Figure 6(a). It can be seen that this is very similar visually to the original reduced size image shown in Figure 6(b) with a significant file size reduction of 107008 bytes.

\section{B. Image Differencing}

The motion activated image is shown in Figure 2 with the Region-of-Interest (RoI) marked as a dotted rectangle and a bird marked with a blue dotted circle. The corresponding difference image is shown in Figure 3. The bird appears as a white blob in the difference image.

The results for the image difference on a DC image are shown in Figure 7. The static and motion-activated images (of size 80x60) are shown in Figure 7(a) and (b). The difference image is shown in Figure 7(c) corresponding to Figure 3. The bird appears as a much smaller blob.

\section{Feature Selection}

After image differencing, two features of interest that can be derived are the blob size and blob count in the difference image (Figure 3 ). The feature values from Figure 3 for the blob count is 1 and blob size is 79 (pixels). 
The size of the blob corresponds directly to the size of the object. The number of blobs could represent a number of objects, or sudden change in lighting conditions. For a spurious event such as camera motion, or an abrupt change in lighting, the blob size and blob count would have very high erroneous values to be realistic and such images could be easily classified as false positives by an intelligent system.

\section{Complexity Reduction}

The operations are reduced considerably. The original parrot image is $768 \times 512$ bytes whereas its DC image shown in Figure 6(a) is only 96x64 bytes.

Assume the image to be of width $w$ and height $h$. If an image pixel processing step requires $w h$ operations, then considering a DC image, the operations reduce to $w h / 64$.

\section{E. Discussion}

In case of applications which do not capture a background image periodically, there would be a requirement to maintain sophisticated background models. There is a trade-off here as for power-constrained applications where it might be desirable to reduce the frequency of capture which might interfere with maintaining a realistic background model. However with static backgrounds and time lapse features such extensive modelling is not required.

The DC coefficients are automatically calculated during JPEG compression, an image standard commonly used for image and video capture in surveillance cameras. The averaging of $8 \times 8$ sub block values as a DC coefficient also smooths out any spurious camera sensor noise improving the results. Using DC coefficients favours long running camera deployments as high resolution images could only be saved for interesting events, thereby increasing the storage time.

The use of features helps with correct object detection as it can reduce false positives. Augmenting the result of background subtraction by taking the blob size and count presents many interesting opportunities. Based on the size of the object to be detected, the thresholds can be appropriately set for both size and count. This would need to take into account the average object size and relating that to its position in the image.

\section{CONCLUSION}

The dumb network edge cameras are being replaced by smart sensor nodes with on-board processing for remote monitoring applications. These intelligent devices can detect an object of interest in the area of operation and autonomously raise an alarm or initiate a response event based on a threshold. By moving the background subtraction algorithms to the camera sensor nodes making them intelligent, results in better resource utilization. DC coefficients for background subtraction methods results in reduced algorithm complexity, prolonging the system's deployment lifetime. Use of simplified and easily extractable features such as blob size and count from image differencing improves the object detection and reduces false positives.
Future work arising from this paper includes using deep learning for object detection to learn features for improved results and reduced occurrences of false positives.

\section{REFERENCES}

[1] S. Nazir, H. Hamdoun, F. Verdicchio and G. Fairhurst, "Autonomous monitoring framework for resource-constrained environments," Cyber-Physical Systems, 2010.

[2] P. L. Rosin, and T. Ellis, "Image difference threshold strategies and shadow detection," in Proc. British Machine Vision Conf.

[3] S. M. Cameron, "Adaptive Sensor Optimization And Cognitive Image Processing Using Autonomous Optical Neuroprocessors," SANDIA REPORT, SAND2001-3304, October 2001.

[4] S. Nazir, S. Newey, Robert Justin Irvine, and Rene van der Wal, "WiseEye: Next Generation Expandable and Programmable Camera Trap Platform for Wildlife Research," January 2017, PLoS ONE 12(1):e0169758.

[5] E. Martínez-Martín, and Á. P. del Pobil, "Motion detection in static backgrounds" Robust Motion Detection in Real-Life Scenarios Part of the series Springer Briefs in Computer Science, pp 5-42.

[6] M. Chakraborty and O. Fuentes, "Real-time Image-Based Motion Detection Using Color and Structure" In: Kamel M., Campilho A. (eds) Image Analysis and Recognition. ICIAR 2009. Lecture Notes in Computer Science, vol 5627. Springer, Berlin, Heidelberg.

[7] P. Jodoin, J. Konrad, V. Saligrama and V. Veilleux-Gaboury, "Motion Detection with an Unstable Camera," 15th IEEE International Conference on Image Processing, San Diego, CA, 2008, pp. 229-232.

[8] M. A. Marzouk, "Modified background subtraction algorithm for motion detection in surveillance systems," AMARABAC, Journal of American Arabic Academy for Sciences and Technology, vol. 1, no. 2, (2010), pp. 112-123.

[9] G. Cocorullo, P. Corsonello, F. Frustaci, L. Guachi-Guachi, and S. Perri, "Multimodal background subtraction for high-performance embedded systems," Journal of Real-Time Image Processing, pp 117, 2016.

[10] T. Yoshida, "Background differencing technique for image segmentation based on the status of reference pixels," International Conference on Image Processing, ICIP '04., Singapore, 2004, pp. 3487-3490, vol. 5, 2004.

[11] A. I. Dell, J. A. Bender, K. Branson, I. D. Couzin, G. G. de Polavieja, L.P.J.J. Noldus, A. Pérez-Escudero, P. Perona, A.D. Straw, M. Wikelski, U. Brose "Automated image-based tracking and its application in ecology" Trends in Ecology and Evolution, vol. 29, issue 7, pp. 417-428, Jul 2014.

[12] S. Khairunniza-Bejo, M. Petrou and A. Ganas, "Local similarity measure for landslide detection and identification in comparison with the image differencing method," International Journal of Remote Sensing, 31:23, 6033-6045, 2010.

[13] A. Sobral, and A. Vacavant, "A comprehensive review of background subtraction algorithms evaluated with synthetic and real videos," Computer Vision and Image Understanding, vol. 122, May 2014, pp. 4-21.

[14] BMC: https://sites.google.com/site/backgroundsubtraction/testsequences

[15] R. A. Bindschadler, T. A. Scambos, H. Choi, and T. M. Haran, "Ice sheet change detection by satellite image differencing" Remote Sensing of Environment, vol. 114, issue 7, 15 July 2010, pp. 13531362.

[16] D. Shah, A. Azari, T. Ko, S. Ahmadian, M. Rahimi, and D. Estrin, "Motion Based Bird Sensing Using Frame Differencing and Gaussian Mixture," available online: https://ssp.ucr.edu/files/DeepShah.pdf .

[17] M. U. K. Khan, A. Khan, and C. M. Kyung, "EBSCam: Background Subtraction for Ubiquitous Computing," in IEEE Transactions on Very Large Scale Integration (VLSI) Systems, vol. 25, no. 1, pp. 3547, Jan 2017.

[18] Widyawan, M. Ihsan Zul, and L. E. Nugroho "Adaptive Motion Detection Algorithm using Frame Differences and Dynamic Template Matching Method," $9^{\text {th }}$ International Conference on Ubiquitous Robots and Ambient Intelligence (URAI), 2012, pp. 236-239.

[19] H. Liu, J. Dai, R. Wang, H. Zheng and B. Zheng, "Combining background subtraction and three-frame difference to detect moving 
object from underwater video," OCEANS 2016 - Shanghai, Shanghai, 2016, pp. 1-5.

[20] P. Terletzky, and R. D. Ramsey, "A Semi-Automated Single Day Image Differencing Technique to Identify Animals in Aerial Imagery," PLOS ONE, 9(1), 2014.

[21] A.Manzanera, and J. C. Richefeu, "A robust and computationally efficient motion detection algorithm based on Sigma-Delta background estimation," Pattern Recognition Letters, Elsevier, 2007, 28(3), available online: http://perso.enstaparistech.fr/ manzaner/Publis/icvgip04.pdf.

[22] J. L. P. Tack, B. S. West, C. P. McGowan, S. S. Ditchkoff, S. J. Reeves, et al., AnimalFinder: A semi-automated system for animal detection in time-lapse camera trap images. Ecol Inform, vol. 36, Nov 2016, pp. 145-151.
[23] P. Dickinson, R. Freeman, S. Patrick, and S. Lawson, "Autonomous Monitoring of Cliff Nesting Seabirds using Computer Vision," In: International Workshop on Distributed Sensing and Collective Intelligence in Biodiversity Monitoring, Dec 2008, Amsterdam.

[24] J. Meng, Y. Juan, and S. Chang, "Scene Change Detection in a MPEG Compressed Video Sequence," IS\&T/SPIE Symposium Proceedings, vol. 2419, February 1995, San Jose, California.

[25] JPEG: https://jpeg.org/

[26] h264: https://www.itu.int/rec/T-REC-H.264.

[27] Nazir, Sajid (2016): WiseEye Trials - Day 3. figshare. Fileset.WiseEye https://doi.org/10.6084/m9.figshare.4129044.v1 